\title{
Optical Microdiscus Resonators
}

\author{
Michalis N. Zervas, G. Senthil Murugan, James S. Wilkinson \\ Optoelectronics Research Centre, University of Southampton, Southampton, SO17 1BJ, UK \\ mnz@orc.soton.ac.uk
}

\begin{abstract}
We present a new "soften-and-squash" method for turning microspheres into high quality, stand-alone microdiscus resonators. Due to the annealing involved in the fabrication process, their measured $\mathrm{Q}$ factors are one order of magnitude better than the starting microspheres. OCIS codes: (140.4780) Optical resonators; (140.3948) Microcavity devices; (230.3990) Micro-optical devices;
\end{abstract}

\section{Introduction}

The last two decades have witnessed huge growth in research activities in theoretical and experimental studies of optical microresonators of different shapes and performance characteristics [1]-[8]. Based on their performance a large number of diverse applications have also been proposed [9]. Among the most extensively studied microresonators are dielectric microspheres, which are easily manufactured with high $Q$ and are versatile in terms of materials and dopants. However, their efficient integration in functional devices remains challenging. The problem becomes even more severe as non-ideal microspheres show a preferred but not easily identifiable plane of resonance and special care is needed to achieve the required optical alignment with the interfacing optics [10]. To overcome some of these problems, highly non-spherical toroidal microcavities have been proposed and studied [3]-[4]. Toroid [3] and microtorus [4] resonators are attached to fibers or vertical stems, respectively, and extra laborious steps are required in order to make them integratable to more functional devices [11].

In this paper, we demonstrate a novel "soften-and-squash" technique for controllably deforming optical microspheres in order to fabricate high performance optical microdiscus resonators. The new resonators belong to the microtoroid group and show (a) higher Q than the original microspheres, due to improved surface quality, (b) easy handling and alignment due to the substantially flattened shape and (c) fewer, more clearly defined resonances due to the pronounced asphericality. The new microresonators have a distinctive discus shape, resembling those described in [5] but about two orders of magnitude smaller in size.

\section{2. "Soften-and-Squash" Microdiscus Fabrication Method}

Microdiscus resonators were fabricated by annealing glass microspheres close to their softening temperature and pressurizing them between two polished glassy carbon plates. While the surface tension of the softened glass is responsible for the discus shape at the circumference of the fabricated microdiscus, the softening temperature and time, as well as the pressure applied on the top carbon plate, determine the thickness of the microdiscus. A microdiscus can also be fabricated to a predefined thickness by having stoppers of specific height on the bottom plate, provided the temperature is sufficient to squash the microsphere up to the stoppers. Figure 1(a) and (b) show a schematic of the fabrication process and a microdiscus fabricated in this way, respectively. In (b) we have also included the fitted microdiscus shape (dotted line) used for the modelling shown below. The initial demonstration used soda-lime silicate glass microspheres as the starting material but the technique is versatile and can be used with any glass material.

(a)
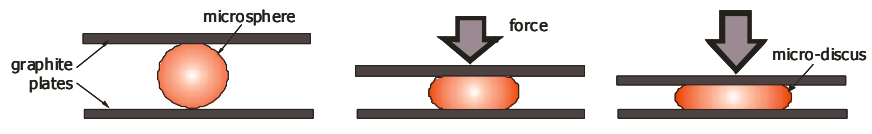
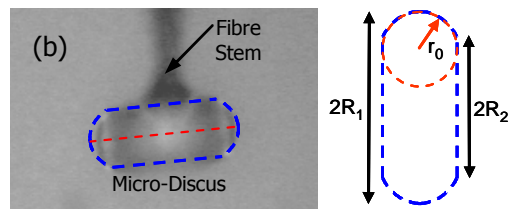

Figure 1: (a) Schematic of the "soften-and-squash" microdiscus fabrication technique, (b) fabricated microdiscus attached to a tapered optical fiber, with fitted shape (blue dashed line).

\section{Resonance Measurements and Discussion}

A soda-lime silicate glass microdiscus fabricated as described above was attached to a tapered optical fiber as shown in Figure 1(b) in order to manipulate the microdiscus while coupling with a waveguide to evanescently excite the WGMs. The microdiscus was positioned on top of a BK7 ion-exchanged planar waveguide using a 3-axis micropositioning stage with the help of the attached tapered fiber stem. Light from a narrow-line tunable laser source was coupled into the waveguide in the TE or the TM polarization using a polarization maintaining single mode fiber. Light scattered from the microdiscus was captured by an InGaAs detector and recorded as a function of 
wavelength, in order to identify the various resonances. Optimized coupling was achieved by controlling the vertical separation using a thin Teflon film over-cladding $(\sim 460 \mathrm{~nm})$ on the waveguide [10].

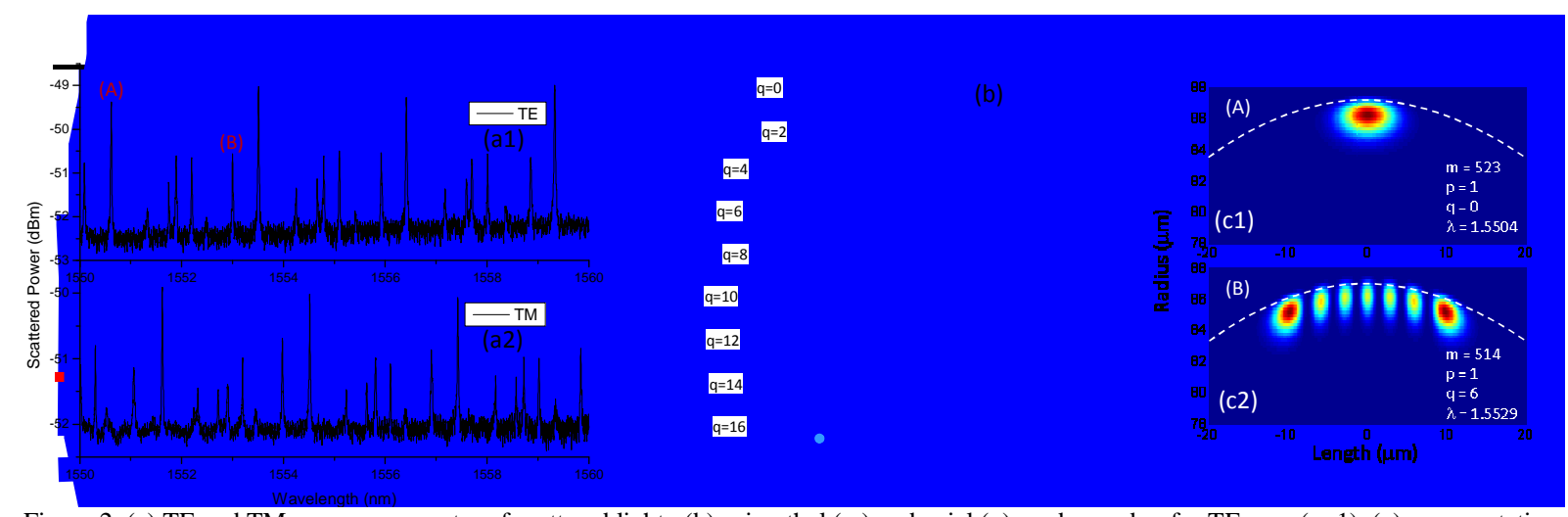

Figure 2: (a) TE and TM resonance spectra of scattered light, (b) azimuthal (m) and axial (q) mode number for TE case (p=1), (c) representative mode intensity profiles.

Figure 2(a) shows the polarization resolved resonance spectra of the microdiscus coupled to the planar waveguide. The measured Q-factors exceeded $10^{5}$ (about one order of magnitude larger than that of the original microsphere). Figure 2(b) shows the predicted TE-polarization resonant wavelengths and the associated azimuthal (m) and axial (q) mode numbers (radial mode number $\mathrm{p}=1$ ). Superimposed are the corresponding experimental data. Figures $2(\mathrm{c} 1)$ and (c2) show calculated intensity profiles for modes $(\mathrm{A})$ and $(\mathrm{B})$ characterised by mode numbers $(m, q, p)=$ $(523,0,1)$ and $(514,6,1)$, respectively. These calculations were based on a newly developed theory based on a transfer-matrix approach and applicable to microresonators with cylindrical symmetry and arbitrary profile [12]. The microdiscus dimensions were obtained from fitted optical microscope images (blue dashed line in Fig. 1(b)) and estimated to be $R_{1}=87.25 \mu \mathrm{m}, \mathrm{R}_{2}=65 \mu \mathrm{m}$ and $\mathrm{r}_{0}=47 \mu \mathrm{m}$. The calculated FSR was $2.902 \mathrm{~nm}$, in excellent agreement with the $2.9 \mathrm{~nm}$ average experimental value. The calculated TM-TE wavelength shift (2.05nm) was slightly larger than the experimental value $(1.9 \mathrm{~nm})$. No special storage precautions were taken for the microdiscus and as humidity is known to result in birefringent stressy layers on the surface of exposed fibers [13], this may have contributed to the measured differences. Similar discrepancies have been observed in polarization splitting in glass microsphere lasers [14]. The TM polarization calculated resonant wavelengths and mode numbers are not shown here.

\section{Summary - Conclusions}

In conclusion, we have presented a new "soften-and-squash" method for transforming microspheres into high quality, stand-alone microdiscus resonators. Due to the inherent annealing involved in the fabrication process, their free surface improved considerably and the measured Q factors were one order of magnitude better than the starting microspheres. Such resonators have a readily identifiable power-confinement plane and are much easier to interface with other optics and integrate into more functional optical devices. In addition, their integration does not require extra laborious steps as in the case of microtoroids [11]. These microdiscus resonators are readily self-aligned with equal-thickness counterparts and can be straightforwardly assembled into laterally- or even vertically-coupled arrays with enhanced properties and functionality such as high-order filters, lasers and photonic molecules. Finally, the proposed fabrication technique can readily produce passive or active chaotic resonators.

\section{References}

[1] K. J. Vahala, "Optical Microcavities", Nature 424, pp. 839 (2003).

[2] V.B. Braginsky, et al, "Quality-factor and nonlinear properties of optical whispering-gallery modes", Phys Lett. A 137 393 (1989).

[3] D.K. Armani, T.J. Kippenberg, S.M. Spillane and K.J. Vahala, "Ultra-high Q toroidal microcavity on a chip", Nature, 421, 925 (2003).

[4] V.S. Ilchenko, et al, "Microtorus: a high finesse microcavity with whispering gallery modes", Optics Letters, 26, 256 (2001).

[5] A.A. Savchenkov, et al, "Kilohertz optical resonances in dielectric crystal cavities", Physical Review A 70, 051804(R), (2004).

[6] G. S. Murugan, et al, "Selective excitation of whispering gallery modes in a novel bottle microresonator", Opt. Express 17, 11916 (2009).

[7] G. S. Murugan, et al, "Hollow-bottle optical microresonators", Opt. Express 19, pp. 20773 (2011).

[8] M. Sumetsky et al "Microbubble resonator", Opt. Lett. 35, 898 (2010).

[9] A.B Matsko, et al, "Review of applications of whispering-gallery mode resonators" IPN Progress Report 42 (2005).

[10]G.S. Murugan et al, "Position-dependent coupling between a channel waveguide and a distorted microsphere resonator", J. Appl. Phys. 107, 053105 (2010).

[11]M. H. Zadeh and K. J. Vahala, "Free ultra-high-Q microtoroid: a tool for designing photonic devices", Opt. Express 15, 166 (2006).

[12]M. N. Zervas, "Transfer-Matrix Analysis of Optical Microresonators", unpublished.

[13]J. Clowes, et al, "Effects of High Temperature and Pressure on Silica Optical Fiber Sensors", IEEE Photonics Tech. Lett.10, 403 (1998).

[14] G.S. Murugan et al, “Integrated Nd-doped Borosilicate Glass Microsphere Laser”, Opt. Lett. 36, 73 (2011). 